\title{
Why New Neurons? Possible Functions for Adult Hippocampal Neurogenesis
}

\author{
Gerd Kempermann ${ }^{1,2}$ \\ ${ }^{1}$ Research Group VolkswagenStiftung at the Department of Experimental Neurology, Charite University Hospital, \\ Humboldt University Berlin, 10117 Berlin, Germany, and 2Max Delbrück Center for Molecular Medicine, Berlin-Buch, \\ 13125 Berlin, Germany
}

The dentate gyrus of the adult hippocampal formation generates neurons throughout life. To date, it remains unclear why. What are the new neurons used for? How can an existing functional neural network integrate and even actively recruit new neurons? The prevailing theories of cognition are based on the assumption that the adult brain is a stable network with regard to the number of neurons. In the current view, structural neural plasticity occurs only at the level of synapses, dendrites, and neurites. The clear demonstration of adult hippocampal neurogenesis, the generation of new granule cell neurons from resident neuronal stem or progenitor cells and their integration in the trisynaptic circuit of the hippocampus, has called this assumption into question. In the light of data on the activity-dependent regulation of adult hippocampal neurogenesis, some conclusions can be drawn, why and how new neurons might contribute to hippocampal function. Our hypothesis is that new neurons do not add memory, but insert strategically "new gatekeepers" at the "gateway to memory".

In hindsight, there is a clear line from the groundbreaking work by Nottebohm (1981), Goldman and Nottebohm (1983), and Barnea and Nottebohm (1996) on functionally regulated neurogenesis in the vocal nucleus and the hippocampus of songbirds to the analogous findings in mammals. However, for the researchers studying neurogenesis in the adult mammalian hippocampus, structural questions came before functional considerations. Only after several studies had confirmed the early reports by Altman and Das (1965) and Kaplan and Hinds (1977) that adult hippocampal neurogenesis indeed occurs, and after improved methods had been introduced to quantitatively identify new neurons with relative ease (Kuhn et al., 1996), did questions of function become imminent. The first studies, particularly the work by Bruce McEwen's group, focused on the negative regulators of adult hippocampal neurogenesis (Gould et al., 1992, 1994), especially severe stress (Gould et al., 1997). In contrast, our own findings that living in an enriched environment led to a robust increase in adult hippocampal neurogenesis indicated that adult hippocampal neurogenesis might indeed be regulated in relation to normal behavior (Kempermann et al., 1997, 1998a). Since then a number of studies have extended these findings (Gould et al., 1999b; Nilsson et al., 1999; Van Praag et al., 1999b), and it became evident that the mammalian hippocampus does indeed show an

I thank Fred H. Gage for intensive discussion of the viewpoint expressed in this review and for helpful comments on this manuscript. I also thank Laurenz Wiskott and Ulrich Anders for their contributions that helped to shape the theory presented in this text.

Correspondence should be addressed to Dr. Gerd Kempermann, Max Delbrück Center for Molecular Medicine, Berlin-Buch, Robert-Rössle-Strasse 10, 13125 Berlin, Germany. E-mail: gerd.kempermann@mdc-berlin.de.

Copyright (C) 2002 Society for Neuroscience $0270-6474 / 02 / 220635-04 \$ 15.00 / 0$ activity-dependent regulation of adult neurogenesis similar to that seen in birds. Despite all similarities, however, there are important differences between birds and mammals, and although the considerations regarding the influence of seasons, food storage behavior, and song learning led the way, they cannot be applied directly to mammals. In contrast to some bird species (Barnea and Nottebohm, 1994), adult neurogenesis in mammals, for example, seems to show very limited dependency on seasons (Lavenex et al., 2000). An equivalent to neurogenesis in song learning has not been identified. In addition, the neuroanatomical structures that exhibit neurogenesis in the songbird and adult mammals have not been demonstrated to be strictly analogous. Almost all data on adult mammalian neurogenesis have been derived from laboratory animals, kept under rather artificial conditions not directly related to their natural habitat. Even the enriched environments of our experiments arguably represent much deprived conditions compared with wildlife conditions. The studies in birds, in contrast, have largely been performed in free-ranging birds or birds held in large aviaries. It is possible, if not likely, that in more closely analogous studies between species common mechanisms and general functional components will be discovered.

The question about the function of new neurons in the adult hippocampus is tightly linked to the more fundamental question of the function of the hippocampus in general, and despite the hippocampus being arguably the best studied functional system of the brain, no detailed consensus has been reached. The hippocampus is classically characterized as "the gateway to memory" (Fig. 1a), but it is clear that the hippocampus is not the "hard drive" of the brain. Although it has some capacity for memory storage, this storage is transient, and the function of the hippocampus therefore appears to be to prepare contents for longterm storage in the cortical areas. The term "gateway" implies just this: a structure, through which all information must pass, before it can be memorized. Despite many profound and elaborate theories, no clear mechanism has been revealed regarding what exactly happens to information, when it passes through the hippocampus. It is obvious, however, that the hippocampus represents a bottleneck in processing the information. In a simplified schematic input, pathways from the entorhinal cortex and other regions project to only 250,000 granule cells of the dentate gyrus (in a mouse). Output from the dentate gyrus through the mossy fiber tracts reaches even fewer pyramidal cells in area CA3. From axons of the CA3, output progresses via CA1 and then once again to the cortical regions. Adult hippocampal neurogenesis occurs at exactly the narrowest spot within the three-synaptic circuit. New granule cells are generated, and mossy fibers are the axons of 
A New gatekeepers at the
gateway to memory?

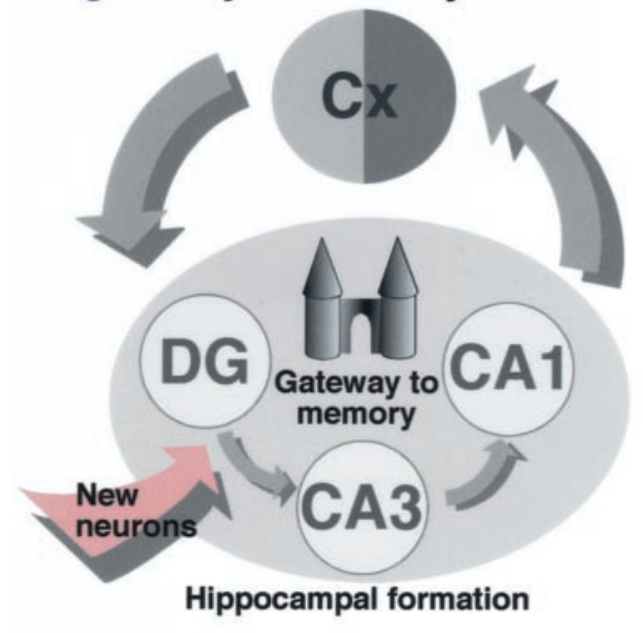

B The simplifying
computer analogy

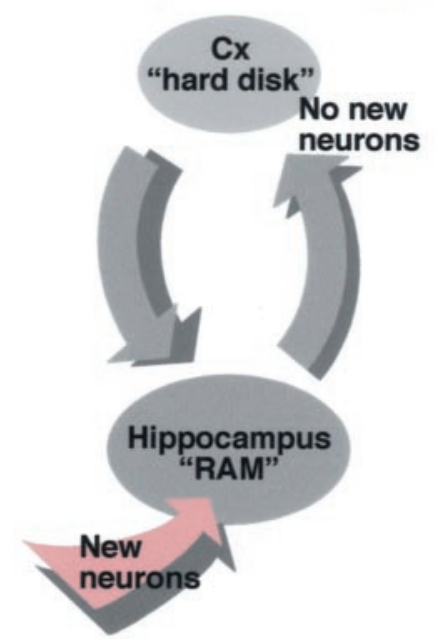

C Strategic addition of new neurons

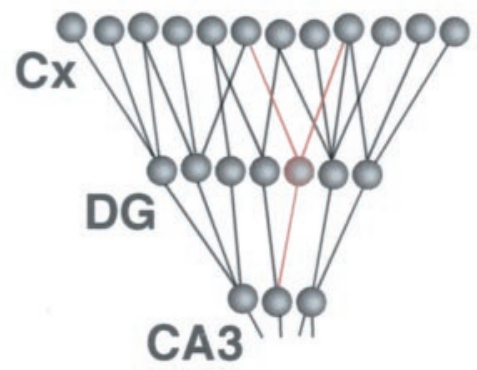

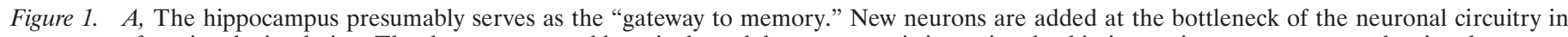

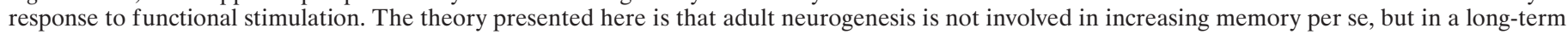

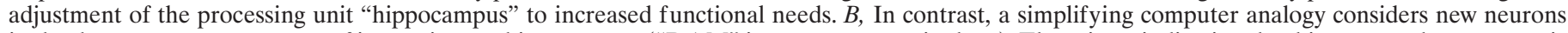

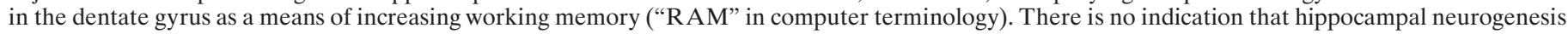

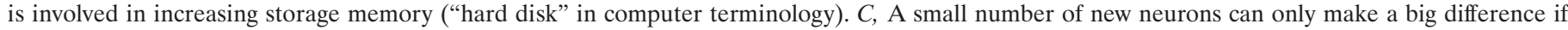
they are strategically used to adapt the existing network. Here, the new neurons are marked in red.

granule cells (Fig. 1a). There is no evidence that memory storage occurs at this level of the circuit. Consequently, we think that the function of adult hippocampal neurogenesis has nothing to do with long-term memory per se. There is no evidence that adult hippocampal neurogenesis equals "putting in more memory, while the computer is running," much less "installing a new hard drive" (Fig. 1b).

If one considers the hippocampus a processing unit, adding neurons here might be more equivalent to an increase in working memory. Even then, however, the small number of new neurons argues against any meaningful "RAM upgrade." In general, there is no evidence that storage of information occurs in single individual neurons. Rather, memory seems to be distributed over the synaptic weights of a network of neurons. Single new neurons, if strategically introduced to a network, might significantly increase in the complexity that can be processed by the network (Fig. 1c).

We have found that the upregulating effects of environmental enrichment on adult hippocampal neurogenesis was paralleled by an improvement on a hippocampal learning task (Kempermann et al., 1997). This is a strong suggestion of a correlation, but no proof of causality. Furthermore, the link between adult hippocampal neurogenesis and learning seems to be bidirectional. Thus, neurogenesis is correlated with experience, and experience effects neurogenesis. It appears that new cells are recruited for function without it being clear to us what this function is. A more isolated learning stimulus alone has been shown to exert such a survival-promoting effect and thus enhance neurogenesis (Gould et al., 1999b), although this result has not been found in other studies with different experimental set-up (Van Praag et al., 1999b). For a discussion of this discrepancy, see Greenough et al. (1999). Still, intuition favors an interpretation that learning can influence neurogenesis.

The question, what the individual new neurons are used for, is difficult to address experimentally. Van Praag et al. (1999a) have shown that long-term potentiation at the level of field potentials, the best known electrophysiological measure of learning, is enhanced in the same mice, in which hippocampal neurogenesis was induced by physical activity. Shors et al. (2001) have treated rats with cytostatic agent methylazoxymethanol acetate (MMA) to reduce hippocampal neurogenesis. They described that the treated animals performed worse than controls on a hippocampusdependent conditioning task, whereas a hippocampus-independent task was spared. Despite adding another piece of suggestive information, this finding can neither prove that new neurons are necessary for hippocampal function nor precisely elucidate the function of the new cells. Although eye-blink conditioning is well known to be a hippocampus-dependent task, one could not say that the function of the hippocampus is mediating eye-blink conditioning. Drugs inhibiting protein synthesis, such as the cytostatic drug MMA, block memory formation, but this effect is presumably independent of effects on cell genesis. In addition, cytostatic drugs tend to have a wide spectrum of effects and side effects, and it is not possible to easily distinguish direct from indirect effects. Intuitively, however, the result of Shors et al. (2001) fit the prevailing theories and are in accordance with earlier results. Thus, the question of what the new neurons actually contribute to hippocampal function remains open.

This functional benefit from adult hippocampal neurogenesis cannot be acute, because it takes several days to generate a functionally integrated new neuron. The new cells extend neurites within a couple of days after division (Hastings and Gould, 1999; Markakis and Gage, 1999), but it is obvious that the new connection cannot benefit the particular functional event, which triggered neurogenesis, because this will long be over when the new neurons are in place. Hippocampal neurogenesis will rather represent a long-term adjustment of the hippocampal circuitry to an experienced level of higher complexity. It would allow the hippocampus to modify the gatekeeper at the gateway to memory and allow a strategic increase in network complexity. This would 
explain why old animals learn quite well, although they have a reduced level of adult hippocampal neurogenesis (Kempermann et al., 1998a). If the new neurons were to expand storage capacity precisely for the one task at hand, this would not make sense. Old animals in a new situation would be in similar need for new neurons as younger ones. If the new neurons, however, represent a refinement of a "processing unit," then it is plausible, why with increasing age, adult hippocampal neurogenesis can decrease without negative functional effects. The overall benefit would not be linked to the one particular stimulus, but would be cumulative. This may be the heart of the question of what we think the use of adult neurogenesis is.

In the context of adult hippocampal neurogenesis, hippocampal function has normally been assessed by testing spatial learning in the water maze task (Morris, 1984), but the water maze task might not be optimal to assess this causality (cf. Eichenbaum, 1996; Eichenbaum et al., 1999; Suzuki and Clayton, 2000). The question is, how far the functions of the rodent hippocampus go beyond spatial memory. A broader functional perspective might, for example, help to explain, why different groups obtained conflicting results when the direct effect of water maze performance on the regulation of adult hippocampal neurogenesis was investigated (Gould et al., 1999a; Van Praag et al., 1999a). "Experience," which is learning in a very broad sense, can stimulate adult hippocampal neurogenesis (Kempermann et al., 1997, 1998a,b; Kempermann and Gage, 1999), but spatial learning might benefit from increased neurogenesis (as seen in our experiment with $129 / \mathrm{SvJ}$; Kempermann et al., 1998b) without being the most relevant factor to stimulate neurogenesis. This idea is supported by the otherwise surprising finding that physical activity causes a dramatic increase in adult neurogenesis and leads to improved performance in the water maze task (Van Praag et al., 1999a,b). Consequently, it will not be sufficient to show that new neurons contribute to learning. It will also be necessary to demonstrate a quantitative correlation between neurogenesis and a functionally (and ethologically) meaningful test of hippocampal function.

From our data we have derived the theory that the function of adult hippocampal neurogenesis is to enable the brain to accommodate continued bouts of novelty. This would fit well with the gateway concept of the hippocampus. The interpretation of adult hippocampal neurogenesis as a mechanism for preparing the hippocampus for processing greater levels of complexity might also explain why the regulation of neurogenesis is linked to physical activity. Physical activity does not per se represent a functional challenge to the hippocampus. Therefore, an upregulation of neurogenesis in this context would not result in any benefit attributable to the stimulus. In contrast to stimulation by environmental enrichment, which led to a survival-promoting effect on the progeny of the proliferating cells in the dentate gyrus, physical activity induced cell proliferation in the subgranular zone. One possible interpretation is that physical activity leads to an increased potential for neurogenesis by inducing stem or progenitor cell proliferation. Learning stimuli could then act on this increased potential by survival and differentiation of the new neurons into functional circuits. It is very difficult to design an experiment that allows sharp distinction between these two forms of stimulation. Neither pure physical activity nor pure learning without any component of physical activity are easily obtainable. Preweaning enrichment is an experimental set-up that exposes very young animals to sensory stimuli during the first 3 weeks of life. This manipulation is known to result in long-lasting effects on cognitive function. However, it did not lead to increased adult hippocampal neurogenesis later in life (Kohl et al., 2002). In contrast, withdrawal from a post-weaning enrichment paradigm had lasting effects at least on the level of cell proliferation (Kempermann and Gage, 1999). One possible interpretation is that the lack of an effect in preweaning enrichment is based on the fact that preweaning enrichment does not involve physical activity or active exploratory pursuit. In this sense, activity might be necessary to elicit the initiation, completion, or maintenance of the neurogenic response.

Taken together, we do not yet know why there are new neurons in the adult brain, but research on regulation of adult neurogenesis, as well as an increasing number of studies directly aimed at elucidating the function of the new cells, have lead us to two conclusions. First, hippocampal neurogenesis is effected by behavior, and in particular behaviors related to hippocampal function. Therefore, difficulties in defining the function of new neurons in the hippocampus also reflects our incomplete understanding of hippocampal function. Second, changes in rate or extent of neurogenesis can have an effect on subsequent behavior. Thus, although behavior can change the structure of the hippocampus, changes in structure can subsequently change or at least effect subsequent behaviors. In a less concrete sense we speculate that the link between activity and adult neurogenesis suggests that new neurons are involved in a general aspect of hippocampal function, most likely sustaining the ability of the dentate gyrus to accommodate the continued modulation of cortical input replete with novel complexities.

\section{REFERENCES}

Altman J, Das GD (1965) Autoradiographic and histologic evidence of postnatal neurogenesis in rats. J Comp Neurol 124:319-335.

Barnea A, Nottebohm F (1994) Seasonal recruitment of hippocampal neurons in adult free-ranging black-capped chickadees. Proc Natl Acad Sci USA 91:11217-11221.

Barnea A, Nottebohm F (1996) Recruitment and replacement of hippocampal neurons in young and adult chickadees: an addition to the theory of hippocampal learning. Proc Natl Acad Sci USA 93:714-718.

Eichenbaum H (1996) Is the rodent hippocampus just for "place"? Trends Neurosci 6:187-195.

Eichenbaum H, Dudchenko P, Wood E, Shapiro M, Tanila H (1999) The hippocampus, memory, and place cells: is it spatial memory or a memory space? Neuron 23:209-226.

Goldman SA, Nottebohm F (1983) Neuronal production, migration and differentiation in a vocal control nucleus of the adult female canary brain. Proc Acad Sci USA 80:2390-2394.

Gould E, Cameron HA, Daniels DC, Woolley CS, McEwen BS (1992) Adrenal hormones suppress cell division in the adult rat dentate gyrus. J Neurosci 12:3642-3650.

Gould E, Cameron HA, McEwen BS (1994) Blockade of NMDA receptors increases cell death and birth in the developing rat dentate gyrus. J Comp Neurol 340:551-565.

Gould E, McEwen BS, Tanapat P, Galea LAM, Fuchs E (1997) Neurogenesis in the dentate gyrus of the adult tree shrew is regulated by psychosocial stress and NMDA receptor activation. J Neurosci 17:2492-2498.

Gould E, Tanapat P, Hastings NB, Shors TJ (1999a) Neurogenesis in adulthood: a possible role in learning. Trends Cogn Sci 3:186-192.

Gould E, Beylin A, Tanapat P, Reeves A, Shors TJ (1999b) Learning enhances adult neurogenesis in the hippocampal formation. Nat Neurosci $2: 260-265$.

Greenough WT, Cohen NJ, Juraska JM (1999) New neurons in old brains: learning to survive? Nat Neurosci 2:203-205.

Hastings NB, Gould E (1999) Rapid extension of axons into the CA3 region by adult-generated granule cells. J Comp Neurol 413:146-154.

Kaplan MS, Hinds JW (1977) Neurogenesis in the adult rat: electron microscopic analysis of light radioautographs. Science 197:1092-1094.

Kempermann G, Gage FH (1999) Experience-dependent regulation of adult hippocampal neurogenesis: effects of long-term stimulation and stimulus withdrawal. Hippocampus 9:321-332.

Kempermann G, Kuhn HG, Gage FH (1997) More hippocampal neurons in adult mice living in an enriched environment. Nature 386:493-495. 
Kempermann G, Kuhn HG, Gage FH (1998a) Experience-induced neurogenesis in the senescent dentate gyrus. J Neurosci 18:3206-3212.

Kempermann G, Brandon EP, Gage FH (1998b) Environmental stimulation of $129 / \mathrm{SvJ}$ mice results in increased cell proliferation and neurogenesis in the adult dentate gyrus. Curr Biol 8:939-942.

Kohl Z, Kuhn HG, Cooper-Kuhn CM, Winkler J, Aigner L, Kempermann G (2002) Preweaning enrichment has no lasting effect on adult hippocampal neurogenesis in four-month old mice. Genes Brain Behav $1: 46-54$.

Kuhn HG, Dickinson-Anson H, Gage FH (1996) Neurogenesis in the dentate gyrus of the adult rat: age-related decrease of neuronal progenitor proliferation. J Neurosci 16:2027-2033.

Lavenex P, Steele MA, Jacobs LF (2000) Sex differences, but no seasonal variations in the hippocampus of food-caching squirrels: a stereological study. J Comp Neurol 425:152-166.

Markakis E, Gage FH (1999) Adult-generated neurons in the dentate gyrus send axonal projections to the field CA 3 and are surrounded by synaptic vesicles. J Comp Neurol 406:449-460.
Morris R (1984) Developments of a water-maze procedure for studying spatial learning in the rat. J Neurosci Methods 11:47-60.

Nilsson M, Perflilieva E, Johansson U, Orwar O, Eriksson P (1999) Enriched environment increases neurogenesis in the adult rat dentate gyrus and improves spatial memory. J Neurobiol 39:569-578.

Nottebohm F (1981) A brain for all seasons: cyclical anatomical changes in song control nuclei of the canary brain. Science 214:1368-1370.

Shors TJ, Miesegaes G, Beylin A, Zhao M, Rydel T, Gould E (2001) Neurogenesis in the adult is involved in the formation of trace memories. Nature 410:372-376.

Suzuki WA, Clayton NS (2000) The hippocampus and memory: a comparative and ethological perspective. Curr Opin Neurobiol 10:768-773.

Van Praag H, Kempermann G, Gage FH (1999a) Running increases cell proliferation and neurogenesis in the adult mouse dentate gyrus. Nat Neurosci 2:266-270.

Van Praag H, Christie BR, Sejnowski TJ, Gage FH (1999b) Running enhances neurogenesis, learning and long-term potentiation in mice. Proc Natl Acad Sci USA 96:13427-13431. 